\title{
Prospect theory and investment decision behavior: A review
}

\author{
Wen Wan $^{1, a}$ \\ ${ }^{1}$ Xi an gao xin NO.1 high school, Shan'xi 510000, China. \\ a1224786002@qq.com
}

Keywords: Investment decision; value function; prospect theory.

\begin{abstract}
In the stock market, influenced by information acquisition, risk preference and other factors, investors' investment decision and behaviors are difficult to be fully rational, so the "rational person" assumption of traditional finance theory is not realistic. Based on behavioral economics, this paper expounds the theoretical basis of prospect theory and explains the irrational investment decision behavior of investors in stock market based on prospect theory. Finally, the paper concludes the investment decision-making frame based on prospect theory. The study of prospect theory can explain investor's emotion and irrational decision-making, and then analyze the investor's investment decision behavior.
\end{abstract}

\section{Introduction}

According to Efficient Market Theory, the stock market is unpredictable. However, behavioral finance research shows that stock returns have a certain degree of predictability. In the strict assumption of the "rational person", the traditional financial theory describes the relationship between the stock prices and their influencing factors with a clear mathematical expression. However, investors are not completely rational, "rational person" assumption is not realistic. Therefore, by using traditional financial theory to predict the stock prices, the effect will naturally not be too ideal. Stock market is a highly complex nonlinear dynamic system. Whether the economic operation is good or not, the fluctuation of the market, the changes of policy and even a conversation of a key person are likely to have a greater impact on the stock market. So using the traditional statistical time series model to predict the stock price naturally will not achieve satisfactory results [1].

In the stock market, once obtaining a small amount of profit, many people will sell the stock, or they will be long-term holders. This part of the people is the majority of individual investors, namely, commonly known as retail investors. A large part of their behavior is psychology at work. Once obtaining a small amount of profit, they do not want to take the risk of waiting for greater profits, or, they will desperate and expect recovery. Here we need to explain the use of prospect theory of behavioral economics. Based on behavioral economics, this paper expounds the theoretical basis of prospect theory and explains the irrational investment decision behavior of investors in stock market based on prospect theory, and concludes the investment decision frame based on prospect theory.

\section{The Theoretical Foundation of Prospect Theory}

Kahneman and Tversky proposed prospect theory in 1979, which proves investors are not entirely rational in the form of experiments [2]. Investors often have incomplete information. In addition, because of the individual differences, the judgement to information of investors will vary depending on subjective value and subjective probability. Finally, investors will deviate from the expectation model of traditional economics, showing irrational characteristics. Nevertheless, this "irrational" has its own law. Anyone who grasps the law can analyze the psychology of investors to predict the stock market [3].

Prospect theory is a perfect combination of psychology and economics, the theoretical basis of which is three deviations and three effects. In cognitive aspect, prospect theory puts forward three 
cognitive biases for the cognitive law in the condition of investor risks. First, the representative bias. In the understanding of new things, people often describe the new business through the similar old things. For example, for a new thing B, people can think of the similar old thing A and will compare the similarity of B and A. By comparing the data, the properties of B can be determined. Second, the availability bias. Due to cognitive ability and memory, people's abilities to obtain information are limited. In the process of decision-making, people only tend to pay attention to easily accessible information and often ignore the other information influencing the decision-making process. As a result, the human intuition will appear to deviate. Third, the anchoring effect. In general, in the process of information processing, people often choose a reference point as the basis of their own decision-making. In the evaluation process, people's decision will be different from the reference point chosen. The reaction deviation and the decision deviation caused by the difference of reference points is called anchor chain effect. Once people's attention is at a certain point, it will affect people's judgments, and thus has a decisive impact on the entire process of decision-making.

The three deviations focus on the process of information collection and processing, while the three effects focus on people's judgment process. Three effects refer to: 1) Certainty effect. People will pay too much attention to confirmed results, giving them higher weights. In other words, in the face of profit, people will show a risk aversion, giving certainty results higher weights, which leads to underestimating the non-deterministic results. The expected utility at this time is not a simple linear expected value. The expecting functions are different on both sides of the reference point, which directly violates the axiom of substitution. Kahneman and Tversky designed a simple experiment to detect the discussion above. Assume that $A$ means 1000 yuan can be obtained 100 percent of the probability, $B$ means it can obtain 1,500 yuan 80 percent of the probability. According to expected utility theory, if the utility of $B$ is 1200 , which is higher than $A$, the rational decision maker should choose $B$, but 82 percent of the people chose $A$. This shows that decision-makers will give the confirmed income a higher weight and neglect the non-deterministic income. 2) Reflection effect. It refers to the opposite of the people's preference when faced with profit and loss. In the positive range of profit, the performance is risk aversion; in the negative range of loss, the performance is risk pursuit. Reflection effect and the deterministic effect is the corresponding. KT added the experiment above into here, but changed the income for the loss. The $A$ represents 100 percent probability of the loss of 1,000 yuan and the $B$ represents 80 percent probability of loss of 1,500 yuan. At last, ninety-two percent of the participants opted for $B$. This shows when decision-makers are dealing with loss and profit, the attitudes to risks are completely different. 3) Isolation effect. When people compare different possible outcomes, they often separate the choice objects first, leaving the common parts and focusing on the different parts of selected objects. Decomposition is in a variety of forms so that different forms of decomposition will lead to the inconsistency between investor preferences and the final choices. Based on the theory of expected utility, the utility is only concerned with the final state of the event. However, the existence of separation effect overcomes the conclusion of the theory of expected utility.

\section{Investment Decision Behavior}

Based on the theory above, Kahneman and Tversky believe that people's risk decision-making often goes through two phases: editing phase and evaluating phase.

In the editing phase, investors collect information, abstract and generalize the information to determine the appropriate decision reference point. The main role of the editing phase is determining the appropriate decision reference point by collecting and arranging the decision-making information, and describing the various options according to certain standards, and then abstracting the decision problem by the standards of reference point. If the result of the decision is better than the reference point, the gain is encoded, otherwise the loss. The editing phase mainly consists of coding, combination, segregation, cancellation, simplification, and dominant checking. 
(1) Coding. Kahneman and Tversky pointed out that decision-makers are concerned about the benefits and losses when making decisions. The reference point in the mind of the investor measures the benefit or loss. However, the position of it is generally related to the current asset level of the decision maker, of course, and related to the way in which information is acquired. The process of coding is the process of describing information in abstract forms. For example, a coin toss. If the front can profit 10 yuan, otherwise you will have to lose 8 yuan. This event can be encoded as $(10,0.5 ;-8$, $0.5)$.

(2) Combination. The process of combination is actually a process of information simplification. It can combine the same attributes in the event for description, making it more concise to state the problem. For example, combining the prospects of $(100,0.3 ; 100,0.25)$ into $(100,0.55)$.

(3) Segregation. Segregation corresponds to combination. It refers to dividing the expectation into the identified part and the risk part according to the probability. For example, in the face of (400, 0.2 ; $300,0.8$ ), the probability of gaining $300 \%$ of the yield is strictly positive, so investors can partition it into risk-free parts, the remaining $(400,0.2)$ is risk component.

(4) Cancellation. Due to the isolation effect, people extract different parts of the expectation and discard the repeated parts.

(5) Simplification. The expectation is simplified by the rounding probability or result. For example, $(99,0.52)$ can be re-encoded for the sake of simplicity to get 100 with a probability of $50 \%$.

The second phase is the evaluating phase. In the evaluating phase, the decision maker evaluates the edited expected value with a subjective measurement and chooses a decision-making plan. After the editing phase, the decision maker evaluates the expectations and chooses the best one. In the evaluating phase, the size of expected value is decided by the value function and the decision weight function together. People will speculate the probability of occurrence of various outcomes when making decisions, and make the right choice through probability reasoning. Traditional finance theory holds that people fully follow the Bayesian rule to make decisions, but the prospect theory holds that in reality, people cannot fully follow the Bayes rule and their behaviors often do not meet the requirements of the ideal model of traditional finance when dealing with uncertainty information. Prospect theory divides probabilities into objective probability and subjective probability. Objective probability is the analysis of physical properties of things, while the subjective probability is people's personal feelings and the judgement of objective probability of the event. In prospect theory, decision weight function is equivalent to probability weight function in expected utility theory. There is a nonlinear relationship between the decision weight and the objective probability, which can be considered as the psychological probability associated with the objective probability.

The value function is the value of subjective feeling of the decision-maker, which is related to the reference point. It is the gain or loss relative to the reference point chosen by the decision-maker, so the measurement of value is the degree of change to wealth rather than the amount of wealth. There is a very important inflection point in the value function called the reference point, which is subjectively determined by the individual as an evaluation criterion. The reference point will change because of the assessment subjects, the environment, and time and so on. In general, the criterion for decision-makers to choose a reference point is the total amount of wealth they now have, but not exactly. After investigating 25 graduate students at Berkeley and Stanford, Kahneman and Tversky used the nonlinear regression method to give the following form of the value function [4]:

$$
v(x)=\left\{\begin{array}{cc}
x^{\alpha}, & \text { if } x \geq 0 \\
-\lambda(-x)^{\beta}, & \text { if } x<0
\end{array}\right.
$$

Among the function, $\alpha, \beta<1, \lambda>1 \alpha, \beta$ are the risk attitude coefficients, and $\lambda$ is the loss aversion coefficient. As can be seen from the equation (1), the profile of value function curve transforms to " $S$ " shape. The curve is concave above the reference point and convex below the reference point. The decision-maker has different attitudes towards risk on both sides of the reference point. The left side of reference point represents the loss, and the right side represents profit. At the time of profit, the decision-maker will increase the risk-averse behavior, performing the early sale of profitable stocks 
in the investment behavior. On the contrary, the investor will prefer the risk when the loss occurs and is unwilling to sell the losing stock. The criterion for determining profit or loss is the reference point at the origin of the value function.

In addition, Rieger found in the study that the value function in the classic prospect theory could not describe the very risk-averse problem in simple sweepstakes. Therefore, he proposed another value function expression, and solved this problem well. The value function expression is as follows [5]:

$$
v(x)=\left\{\begin{array}{cl}
1-e^{-\alpha x}, & \text { if } x \geq 0 \\
-\lambda+\lambda e^{\beta x}, & \text { if } x<0
\end{array}\right.
$$

Four conclusions can be drawn from the summary of the prospect theory: (1) Comparing the amount of change in wealth with the absolute amount of wealth, people are more fancy the former. In other words, people are more concerned about the profit or loss after the investment. (2) When people deal with the prospects of loss, they tend to prefer risks rather than to achieve the loss. While they deal with the prospect of earnings, it is opposite. They tend to avoid risk to achieve certainty of profit. (3) The degrees of sensitivity to the loss and acquisition are different; the sensitivity of the loss is greater than the sensitivity of the acquisition. (4) The results of decision-making will affect investors' attitudes towards risk. Investors will enhance the risk appetite because of pre-earnings, while early loss will weaken the risk appetite of investors. For investors, the utility they gain from the current profit or loss depends on the result of pre-investment. In the prospect theory framework, the risk decision process of the investor is shown in Fig 1.

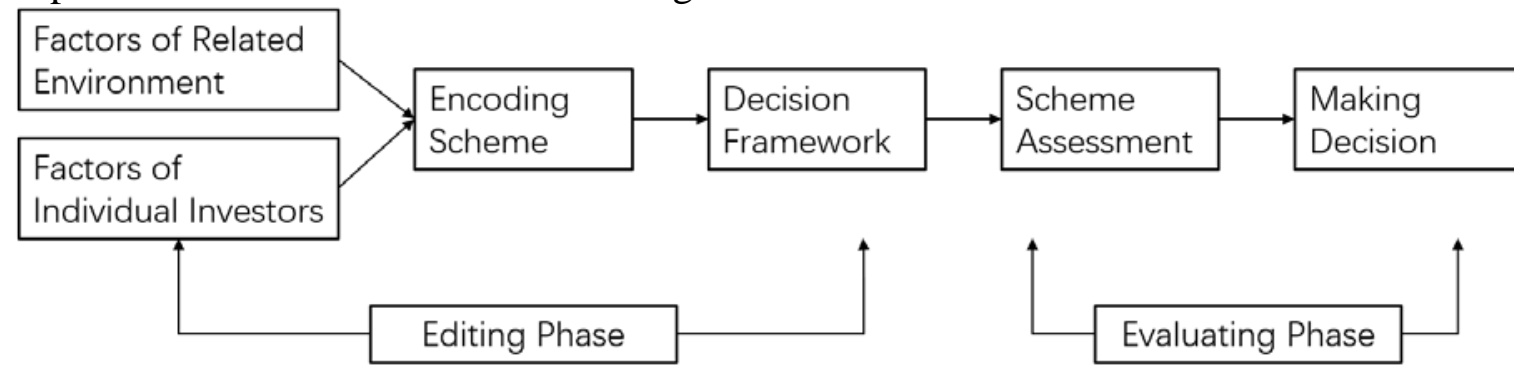

Fig. 1 Investment Decision Framework Based on Prospect Theory

\section{Conclusion}

In this paper, we elaborate the theoretical basis of prospect theory and explain the irrational investment decision behavior of investors in the stock market based on the prospect theory, and obtain the investment decision framework based on prospect theory. When the outcome of the action is benefiting, the actor is a risk averse. While the outcome of the action is having loss, the actor is a risk loving. The value function of prospect theory shows S-type, which shows that investors tend to sell securities first in the profit state instead of taking certain risks. While they tend to hold securities in the red, and tend to bear uncertain risks.

\section{References}

[1] Barberis, N., \& Thaler, R. (2003). A survey of behavioral finance. Handbook of the Economics of Finance, 1, 1053-1128.

[2] Kahneman, D., \& Tversky, A. (1979). Prospect theory: An analysis of decision under risk. Econometrica: Journal of the econometric society, 263-291.

[3] Woolridge, J. R., \& Snow, C. C. (1990). Stock market reaction to strategic investment decisions. Strategic management journal, 11(5), 353-363.

[4] Tversky, A., \& Kahneman, D. (1992). Advances in prospect theory: Cumulative representation of uncertainty. Journal of Risk and uncertainty,5(4), 297-323. 
[5] Rieger, M. O., \& Bui, T. (2010). Too risk-averse for prospect theory?. 